\title{
Modified Negative Airflow Aerosol Prevention Box for COVID-19 Patients
}

\author{
Neeraj Kumar ${ }^{1}$, Abhyuday Kumar $^{2}$, Amarjeet Kumar $^{3}$, Chandni Sinha $^{4}$
}

\begin{abstract}
The importance of this intubation box has come to light recently in view of the COVID-19 pandemic and the fact that intubation is an aerosolgenerating procedure (AGP). Risks of the healthcare worker attending to the airway of COVID-19 patients is high and the intubation box aims to minimize that and reduce contamination of the environment. To address this objective of decreasing transmission during AGP, we created a negative airflow aerosol box with a leak-proof airway handling system using readily available and affordable materials. The dimension of this box was $24 \times 17.5 \times 17.5 \mathrm{~cm}$ and it was made of high-quality $4 \mathrm{~mm}$ transparent acrylic sheet with two arm holes of $10 \mathrm{~cm}$ diameter. The caudal end of the negative airflow aerosol prevention box is wrapped with disposable plastic sheet and the both hand slots are sealed using camera cover and latex hand gloves and it decreases the risk of contamination.

Keywords: Aerosol-generating medical procedures, COVID-19 in India, Emergency medical services, Endotracheal intubation.

Indian Journal of Critical Care Medicine (2020): 10.5005/jp-journals-10071-23633
\end{abstract}

\section{BACKGROUND}

Today, we are in the midst of novel coronavirus disease (COVID19) pandemic and it has not only affected the global social and economic status but has also challenged the entire healthcare system. Aerosol-generating procedures (AGPs) like intubation and extubation have the potential for fugitive emissions and carry a higher risk of transmission of the virus to the surrounding environment and should only be performed when absolutely necessary negative-pressure environments with frequent air exchanges under the care of highly trained personnel. Anesthesiologists by virtue of their closeness to the airway are more prone to infection. Lack of proper personal protective equipment (PPE), prolonged exposure, insufficient spacing, and operating rooms without negative pressure can further multiply the risks. The importance of this intubation box has come to light recently in view of the COVID-19 pandemic and the fact that intubation is an AGP. Risks of the healthcare worker attending to the airway of COVID-19 patients is high and the intubation box aims to minimize that and reduce contamination of the environment. Dr Lai Hsien-yung, an anesthesiologist from Taiwan, first formulated the concept of aerosol box. ${ }^{1}$ Then, Canelli et al. recently invented an "aerosol box" and the efficacy of this box in reducing droplet scatter has been studied in a Mannequin with the simulation of cough. ${ }^{2}$ They invented this box to prevent aerosol-mediated viral infection during intubation/extubation either during emergency or elective procedures in a suspected COVID patients. A negativepressure operating room is suggested to contain this new virulent killer and hospitals are racing with time, budget, and infrastructure to create them.

\section{TeCHNIQUe}

To address this objective of decreasing transmission during AGP, we created a negative airflow aerosol box with a leak-proof airway handling system using readily available and affordable materials. The dimension of this box was $24 \times 17.5 \times 17.5 \mathrm{~cm}$ and it was made of high-quality $4 \mathrm{~mm}$ transparent acrylic sheet with two arm

\footnotetext{
${ }^{1,3}$ Department of Trauma and Emergency, All India Institute of Medical Sciences, Patna, Bihar, India

2,4 Department of Anaesthesiology, All India Institute of Medical Sciences, Patna, Bihar, India

Corresponding Author: Neeraj Kumar, Department of Trauma and Emergency, All India Institute of Medical Sciences, Patna, Bihar, India, Phone: +91 8210104972, e-mail: neeraj.jnmc@gmail.com

How to cite this article: Kumar N, Kumar A, Kumar A, Sinha C. Modified Negative Airflow Aerosol Prevention Box for COVID-19 Patients. Indian J Crit Care Med 2020;24(10):981-982.

Source of support: Nil

Conflict of interest: None
}

holes of $10 \mathrm{~cm}$ diameter. The caudal end of the negative airflow aerosol prevention box is wrapped with disposable plastic sheet and the both hand slots are sealed using camera cover and latex hand gloves and it decreases the risk of contamination. We made multiple small holes in the distal $30 \mathrm{~cm}$ of the intercostal drain tube and fixed it diagonally from inside over the roof of the aerosol box. This intercostal drain tube was then passed through the underwater seal drain system containing $100 \mathrm{~mL}$ of $1 \%$ sodium hypochlorite to disinfect the exhausted air before attaching to the wall suction unit. Inlet for surrounding airflow was provided through space surrounding the entry of the anesthesia circuit (Figs 1A to $C$ ).

\section{Discussion}

As the negative-pressure rooms require a minimum of 12 air changes of exhaust per hour or a flow rate of 145 liters per second per patient, ${ }^{3}$ we kept the suction pressure at 200 mbar using HagenPoiseuille equation to create approximately 12 air changes per hour needed in a 70-liter volume intubation box. We assessed the efficacy of physical barrier and negative airflow through the suction tubing by aerosolization of fluorescein solution under ultraviolet (UV) light. Aerosolization was created within the box using Cirrus nebulizer (Medi Safe International, Delhi, India) and the movement 

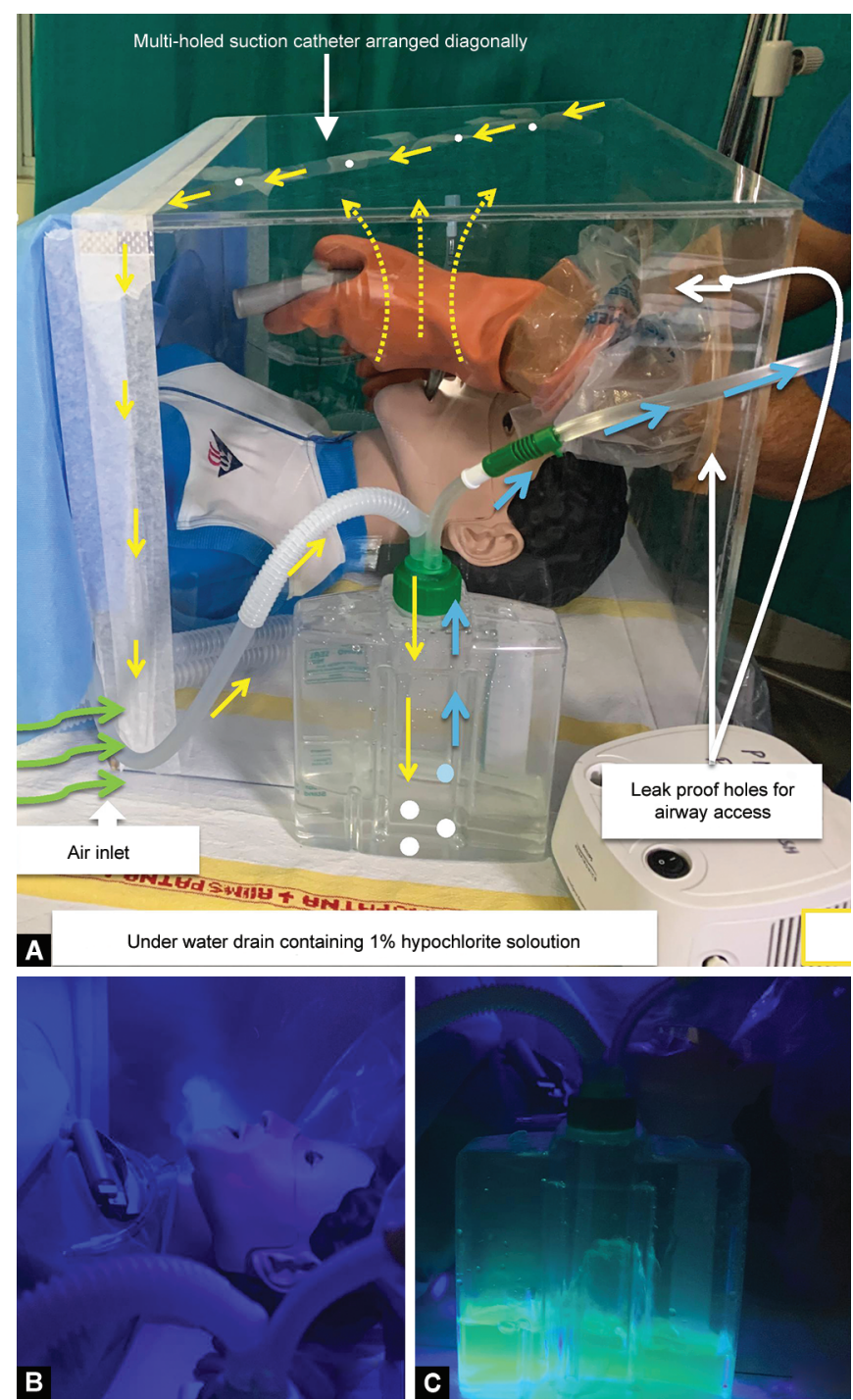

Figs $1 \mathrm{~A}$ to C: (A) Negative airflow aerosol box; (B) Fluorescein aerosolization seen under ultraviolet light; (C) Fluorescent fluid in underwater seal bottle seen in UV light

of aerosolization toward the suction was visible. After 15 minutes of activation of suction, fluid in the underwater seal bottle was seen as fluorescent in UV light indicative of its effectiveness. With this modification, the laryngoscopist's gloves, gowned forearms, face mask, eye shield, hair, neck, ears, and shoes would be prevented from contamination as the box was made leak-proof cranially. Camera cover was attached to inbuilt gloves for preventing leak and to give extra space for physician's range of motion during the procedure. Extra holes with windows can be made on the lateral sides of the box for the emergency passage of hand when laryngeal manipulation, cricoid pressure, or extra help is needed. Dalli et al. ${ }^{4}$ by the use of high-speed monochromatic camera showed that substantial amounts of air moves out of the intubation box from the caudal end as well as the holes on the user side. In our model, we did not see any fluorescein coming over the intubator's face mask or body part, as the cranial working ports were well-sealed using the camera cover and latex hand gloves. The caudal end of the box was wrapped with disposable plastic sheet.

Our technique of using this modified negative-pressure aerosol prevention box is simple and cost-effective that can be readily applied onto the patient, serving to reduce aerosol dispersal during elective and emergency airway management of COVID-19 patients.

The advantages of this modified negative air flow aerosol prevention box are:

- Complete protection from aerosol dispersion during intubation, extubation, and nebulization either in operating room (OR) or intensive care unit (ICU).

- Affordable, safe, and effective method of protection.

- Reusable after decontamination with $70 \%$ alcohol or $1 \%$ sodium hypochlorite spray.

- Useful in regional anesthesia for supplemental oxygen and airway access.

- This device can protect healthcare workers especially in emergency situations or in emergency rooms, where the infection status of the patient is unclear, doctors and nursing staffs are at high risks of exposure.

- Leaks or aerosol exposure can be prevented by making necessary and sufficient cuts and notches all notches are sealed with tape which helped to achieve more airtight conditions.

- The box is leak-proof cranially by the use of camera cover attached to inbuilt gloves to prevent any aerosol leaks from these holes as they are completely sealed.

\section{Limitations}

- This modified negative airflow aerosol box remained untested in human volunteers.

- This box cannot be used in difficult airway.

- The dispersion of fluorescein dye was not quantified.

\section{Conclusion}

We acknowledge that the device is not tested for its effectiveness in preventing the infection. This device is a prototype intended to be used during this period of COVID-19 pandemic where the world is in search of new techniques to counter this virus. However, a better device based on these principles can be engineered in future.

\section{HighLightS}

This modified negative airflow aerosol prevention box for COVID-19 patients consists of transparent box, negative airflow via suction device, leak-proof cranially using camera cover and inbuilt gloves and extra space was provided for physician's range of motion during airway manipulation.

\section{Informed Consent}

Informed consent was not needed.

\section{References}

1. Everington K. Taiwanese doctor invents device to protect US doctors against coronavirus. Taiwan News. March 23, 2020. https://www. taiwannews.com.tw/en/news/3902435.

2. Canelli R, Connor CW, Gonzalez M, et al. Barrier enclosure during endotracheal intubation. N Engl J Med 2020;382(20):1957-1958. DOI: 10.1056/NEJMc2007589.

3. American Society of Anesthesiologists. COVID-19. Information for Health Care Professionals. Available from https://www.asahq. org/about-asa/governance-and-committees/asa-committees/ committee-on-occupational-health/coronavirus. Date last accessed: May 29, 2020.

4. Dalli J, Khan MF, Marsh B, et al. Evaluating intubation boxes for airway management. Br J Anaesth 2020;125(3):e293-e295. DOI: 10.1016/j. bja.2020.05.006. 